\title{
A Brownian Motion technique to simulate gasification and its application to $\mathrm{C} / \mathrm{C}$ composite ablation
}

\author{
J. Lachaud* and G. L. Vignoles \\ University of Bordeaux 1 \\ Laboratoire des Composites ThermoStructuraux (LCTS) \\ 3, Allée de La Boétie, 33600 Pessac, France
}

\begin{abstract}
Ablation of carbon-carbon composites $(\mathrm{C} / \mathrm{C})$ results in a heterogeneous surface recession mainly due to some gasification processes (oxidation, sublimation) possibly coupled to bulk mass transfer. In order to simulate and analyze the material/environment interactions during ablation, a Brownian Motion simulation method featuring special random walk rules close to the wall has been implemented to efficiently simulate mass transfer in the low Péclet number regime. A sticking probability law adapted to this kind of random-walk has been obtained for first-order heterogeneous reactions. In order to simulate the onset of surface roughness, the interface recession is simultaneously handled in 3D using a Simplified Marching Cube discretization. This tool is validated by comparison to analytical models. Then, its ability to provide reliable and accurate solutions of ablation phenomena in 3D is illustrated.
\end{abstract}

Key words: Brownian Motion, Sticking event, Receding surfaces, Carbon-carbon composites

PACS: 87.10.Rt, 05.40.Jc, 81.65.Mq, 68.35.Ct, 68.35.Fx, 72.80.Tm

\section{Introduction}

Carbon/Carbon (C/C) composites, which keep excellent mechanical properties at high temperatures [1], are used as thermostructural protections in various applications such as atmospheric re-entry (vehicle

\footnotetext{
* Corresponding author; Phone number: +1 (650) 265 8474; Fax number: +33 (0) 5568412 25;
} Email address: jean.lachaud@gadz.org 
thermal protection systems) [2], propulsion (rocket nozzle) [3], and experimental Tokamak reactors for nuclear fusion (divertor and first wall armor) [4]. In these applications, the C/C composites are mainly ablated by some gasification processes (oxidation, sublimation) [2]. An efficient design of a thermostructural protection relies on an accurate evaluation of the wall recession caused by ablation. In particular, $\mathrm{C} / \mathrm{C}$ composites develop surface roughness features, which may display a strong coupling with the surrounding environment $[5,6]$. The surface roughness chiefly arises from the heterogeneity of the composites, whose fibers and matrices are made of distinct forms of carbon [7]. In a previous work, the composite behavior has been analytically modeled in steady state [6]. The results were in agreement with the experimental observations [7] and provided a good understanding of $\mathrm{C} / \mathrm{C}$ composite behavior during ablation. Nevertheless, in order to enable an analytical modeling, the composite architecture had been simplified as much as possible. Mainly, as a first approximation, the fiber section had been considered circular, while the actual section outline slightly undulates around a mean circular line. Moreover, a 3D modeling of the transient behavior cannot be attained through purely analytical methods. This paper aims to present a 3D numerical simulation tool implemented to model with more accuracy $\mathrm{C} / \mathrm{C}$ composites and their surface roughening during ablation.

Physico-chemical ablation, studied at microscopic scale, typically belongs to heterogeneous reaction/mass transfer problems with a receding surface $[2,6,8,9]$. The dynamics of nontrivial pattern formation at small scales poses a serious challenge for numerical simulations. A precise integration of the equation of surface motion at the pattern scale is mandatory. For instance, Finite Volumes/Volume-of-Fluid methods represent one possible solution for this type of problems, and are quite efficient for most of the cases [10]; but they may be extremely memory-consuming as soon as large images with possibly complicated patterns are to be dealt with. A multigrid approach may be a solution to this inconvenient, since the gas concentration field some distance away from the surface undergoes small 3D gradients compared to the surface. Another way to tackle this problem is to use a Random Walk description of mass transfer : this method is known to provide excellent results when computing effective transport coefficients, i.e. only an average gas behavior, inside large and complex 3D images of porous media [11]. Random Walk methods are available for the simulation of either continuum [12], transition or rarefied gas transport [13]. However, the algorithms have to be extended in order to account for the heterogeneous reaction. One of the solutions proposed in the past rests on the coupling of the Random-Walk code far from the surface with a Finite-Difference solver close to it [14]. Here it is chosen to address the gas-solid reaction directly through the use of a suited sticking probability law. This represents a lighter algorithm, and allows to handle easily the surface evolution, thanks to a simplification of the Marching Cube method [15] for the 
surface discretization.

In a first section, the mathematical form of the studied problem is presented. In a second section, the numerical tool itself is presented and validated by comparison to the analytical solution obtained in [6] in the case of circular fibers. In the last section, an ablation simulation is carried out on a realistic fiber section and compared to experimental observations of surface roughness features.

\section{Model and analytical solution}

\subsection{Ablation model}

The ablation process leads to the recession of the material surface $S$ under heterogeneous gasification processes, like oxidation or sublimation [2]. The macroscopic motion of this surface can be interpreted as an advancing wavefront. The surface position may be described in cartesian coordinates by the following scalar equation:

$$
S(x, y, z, t)=0
$$

such that the function $S$, which possesses first order partial derivatives almost everywhere, acquires nonzero values at all points not lying on the interface [16]. This equation may be derived with respect to time :

$$
\frac{\partial S}{\partial t}+\mathbf{v} \cdot \nabla S=0
$$

where $\mathbf{v}=v J \mathbf{n}$ is the surface local normal velocity, with $v$ the local solid molar volume of the ablated material [16]. In the case of heterogeneous materials, $v$ varies with space. $J$ (in $m o l . m^{-2} \cdot s^{-1}$ ) is the molar rate of ablation. In this work, it is assumed to follow a first order kinetic law for oxidation and a Knudsen-Langmuir model for sublimation [2,17]. The Péclet mass number is low close to the wall $[7,17]$. Under these hypotheses, oxidation and sublimation are shown to be mathematically equivalent as far as gasification and mass transfer are concerned $[2,17]$. Using oxidation notations, the model writes :

$$
J=-D \nabla C \cdot \mathbf{n}=-k \cdot C(S=0)
$$

where $k$ (in $m . s^{-1}$ ) is the gasification rate of the material (like $v, k$ is a function of space for heterogeneous materials), $D$ (in $m^{2} . s$ ) the diffusion coefficient of the reactant in the bulk fluid phase, and $C(S=0)$ (in mol. $\left.\mathrm{m}^{-3}\right)$ is the reactant gas concentration at the material surface. Mass conservation of the reactant in the fluid writes :

$$
\frac{\partial C}{\partial t}+\nabla \cdot(-D \nabla C)=0
$$


Boundary conditions for concentration relative to the model domain have to be specified (see for example figure 1-a). A Dirichlet condition $\left(C=C_{0}\right)$ on the boundary layer top is shown to be suitable [6]. On lateral boundaries, a periodicity condition is retained. The interface position $S$ as a function of space and time is then given by the simultaneous resolution of equations 2 and 4 . For heterogeneous materials, this model cannot be solved analytically in the general case.

\subsection{An analytical solution}

Let the analytical solution previously obtained for circular fibers [6] be briefly recalled here since it is a perfect reference case for the following 3D validation of a the simulation tool (see section 3.2). The general model of section 2.1 has been applied to the cell represented on figure 1-a. It represents a carbon fiber embedded in a carbon matrix shown before ablation: the surface is initially flat (no surface roughness). Translation conditions on lateral boundaries and the Dirichlet condition $\left(C=C_{0}\right)$ on boundary layer top are imposed. Under these boundary conditions, the cell represents a composite yarn, whose lateral extension is infinite, and ablated perpendicularly to its principal direction. Both components are gasified by a first-order reaction (oxidation of carbon by oxygen for example), the matrix being more reactive than the fibers [7]. The concentration gradient of reactant in the fluid phase has been shown to be vertical in steady state [6], that is, along $\mathbf{z}$ (see figure 1-a). An analytical solution to this problem gives the following results [6,17]: i) Due to the higher recession rate of the matrix, the fibers are partially stripped, become thinner, and acquire a needle shape. ii) Only two parameters are necessary to describe the steady state surface roughness features of the yarn : a reactivity contrast $A$ and a Sherwood number $S h$. The reactivity contrast between the matrix and the fibers $A$ is given by:

$$
A=\frac{k_{m} v_{m}}{k_{f} v_{f}}
$$

where $k_{m}, v_{m}, k_{f}$, and $v_{f}$ are respectively the matrix and fiber reactivities and solid molar volumes, which are assumed constant for each phase. For the standard composites and according to literature data, fiber and matrix densities are of the same order of magnitude, while matrices are neatly more reactive than fibers; hence, one has $A>1$ [7]. The geometry of the steady state is strongly linked to this contrast; the higher it is, the higher the denuded fiber size $h_{f}^{s}$ (from fiber tip to matrix level) is when steady state is reached. The Sherwood number $S h$ writes :

$$
S h=\frac{k_{m} R_{f}}{D}
$$

where $R_{f}$ is the fiber radius. It is an indicator of the three reaction regimes that can appear on this heterogeneous reactive surface : a diffusion-limited regime $(S h>>1)$, a transition regime $(S h \sim 1)$, 
and a reaction-limited regime $(S h<<1)$. For small values of $S h$, the fiber tip geometry is conical and only depends on $A$, with $h_{f}^{s}=R_{f} \sqrt{A^{2}-1}$ (see figure 1-b). When $S h$ increases but is lower than $\sqrt{A^{2}-1}$, diffusional effects tends to lower $h_{f}^{s}$ and the fiber tips become ogival. For higher values of $S h$, fibers tips display a plateau and $h_{f}^{s}=R_{f}(A-1) / S h$. Hence, in diffusion-limited regime, $h_{f}^{s}$ tends towards zero.

This analytical model seems then of great interest for a validation of the numerical simulation tool, as well as for the diffusion and reaction part (all reaction/diffusion regimes are scanned) than for the front tracking because 3D surfaces including singularities (fiber tips) are featured.

\section{Numerical solution}

AMA has been developed on a Monte-Carlo Random-Walk principle [18-23]. AMA is the acronym for: Ablation Marches Aléatoires (Random Walk Ablation in French).

\subsection{AMA algorithm presentation}

A scheme of the general algorithm and a sketch of AMA main features are presented on figures 2-a and b. First, a 3D image containing several phases (fluid/solids) is generated. It is discretized as greyscale values on a 3D cubic grid. Then, the fluid/solid interface (equations 1) is determined by a simplified marching cube approach [20], which is a linear approximation of the actual surface in each voxel (3D pixel) of the interface. Mass transfer from the bulk gas to the wall (equation 4) is simulated by a random walk based on the Brownian motion simulation technique, which is a continuum (grid-free), rapidly converging and memory-sparing method for diffusion in a continuous fluid [18]. In order not to degrade the numerical accuracy of the method, it is most convenient to give a constant representativeness to each random walker (i.e. a constant number of actual gas molecules associated to each walker). In addition, one has to satisfy the Dirichlet boundary condition on the upper part of the fluid phase. This is achieved through the use of a buffer zone in which a constant number of walkers is ensured. The total number of walkers in the rest of the fluid phase results from the diffusion/reaction process, that is, the coupled resolution of equations (3) and (4). As represented on figure 2-b, the path followed by a walker is not the physical one dictated by gas kinetics, but another one based on Brownian motion simulation technique that requires less computational time [18]. One of the properties of Brownian motion is that, in average, the space 
increment $\Delta r$ covered by a molecule is related to the time increment $\Delta t$ by the following equation:

$$
\Delta t=\frac{\Delta r^{2}}{6 D}
$$

where $D$ is the diffusion coefficient. Using this property for simulation purpose, the size of the space increment has obviously to be chosen as large as possible to decrease the computational time, but small enough when compared to the characteristic size of the problem. A parametrical study must then be carried out to determine the relevant space increment; it is the equivalent of a mesh study for finite element methods. The trajectory of each walker is followed individually during a given time interval $\Delta t_{e}=N_{s} \Delta_{t}$, where $N_{s}$ is a chosen number of steps. The value of $\Delta t_{e}$ is evaluated to ensure a walk duration leading to a probability of about $50 \%$ to hit a wall. In general, $N_{s}$ is rather large. At each step, the walk direction is determined randomly and equiprobably in 3D space. The heterogeneous first-order reaction on the wall (equation 3) is simulated using a sticking event based method [19]. Let us assume that a walker hits the surface after a walk duration $\Delta t_{w}\left(0<\Delta t_{w}<\Delta t_{e}\right)$. A random number between 0 and 1 is generated from a uniform distribution. If that number is higher than a previously determined sticking event probability, the walker bounces back and its walk goes on until either it hits the wall again or $\Delta t_{e}$ is reached. In the converse case, the walker is assumed to stick; another walker is released in the buffer zone, for a walk duration of only $\Delta t_{e}-\Delta t_{w}$. This ensures that in steady state conditions the flux received by the wall is equal to the flux entering the simulation box by its upper side.

Every sticking event is associated to the solid node which lies nearest to the exact location where it takes place. The amount of sticking events is recorded for every solid node of the fluid/solid interface. Whenever for a given node the cumulated number of sticking events reaches a given threshold, that is, when the required quantity of reactant to gasify one voxel is reached, it is converted into fluid. Then, the surface is modified and has to be updated (equation 2). When a significant number of solid voxels is turned into fluid, an image is recorded. When an appreciable number of fluid voxels has been created (say, the volume of one horizontal slice of the image), the resolution domain is shifted downwards by the corresponding amount (one voxel height), in order to preserve a constant fluid volume throughout the simulation. The average ablation velocity can be inferred using the frequency of geometry shifts. The average ablation velocity and reactant flux are compared with the value obtained at the previous geometry shift operation. When the flux is constant from one run to another, this means the steady state is reached, and then the simulation ends. The steady state may also be visualized by $3 \mathrm{D}$ rendering and characterized by image analysis. Let some critical steps of the algorithm be further detailed.

To handle accurately the Dirichlet boundary condition, a parameter variation study has shown that the 
size of the buffer zone should be at least twice as large as the space increment $\Delta r$ of the random walk. As sketched on figure 2-b, using a buffer zone, the boundary condition is applied in practice at a position $\Delta r$ inside that zone, the size of the concentration boundary layer $\delta_{c}$ being then obtained by the sum of the fluid phase height (buffer excluded) and $\Delta r$.

The choice of the space increment size is critical as far as accuracy and computational time are concerned. In the bulk fluid phase, $\Delta r$ has to be taken as large as possible, in order to lower the computational time. Conversely, close to a wall, a parameter variation study has shown that $\Delta r$ should be smaller than the characteristic size of the surface roughness (fiber radius in our case for example). Indeed, in the converse case, some zones may be numerically shaded. Moreover, some conditions have to be respected for the walker diffuse reflection to insure mass conservation. When a walker hits the wall, its last space increment $\Delta r_{w}$, which leads to collision, is smaller than $\Delta r$ (or at most equal). In this algorithm, the walker bounces back according to a diffuse emission law (Knudsen's cosine law) with a space increment $\Delta r_{w}$. Using this bouncing rule, the average space increment size close to the wall automatically decreases, leading to a more accurate simulation of surface recession. Compared to finite element, this is the equivalent of an adaptative mesh refinement that follows the wall recession.

The heterogeneous first-order reaction on the wall is simulated by a sticking event with a probability $P_{s}$ adapted to the Brownian motion simulation technique. By definition, the sticking probability is the ratio between the number of molecules that are consumed (or stuck) on the wall and the number of molecules hitting the wall. In term of molar fluxes, this writes [19]:

$$
\tilde{P}_{s}=\frac{\Phi_{R}}{\Phi_{M T}+\Phi_{M A}}
$$

$\Phi_{R}=C k$ is the molar flux consumed on the wall. $\Phi_{M T}$ is the molar flux transported to the wall by mass transfer, which is equal to $\Phi_{R}$ in steady state (mass conservation). In the case of ablation or condensation, the solid surface velocity is slow compared to mass transport because of the low solid to gas molar volume ratio; hence, surface evolution and mass transport are decoupled and mass transport can be considered as a steady state phenomenon. $\Phi_{M A}$ is the molecular agitation flux. In the case of random walks following Maxwell distribution, this flux is called half Maxwellian flux and writes $1 / 4 C\langle v\rangle$, where $\langle v\rangle$ is the molecular agitation mean velocity. The numerical velocity of a walker hitting the wall is $v=$ $6 D / \Delta r_{w}$ (from eq. 7). The mean quadratic length $\left\langle\Delta r_{w}\right\rangle$ is potentially a function of the surface roughness or the presence of notches. Therefore, to maintain the accuracy of the simulations in the case of complex geometries, it is important to keep $\Delta r_{w}$ itself in the numerical method, the mean quadratic length being computed during the simulation. To account for the fact that the Brownian Motion random walk 
implemented does not follow a Maxwellian distribution law, the introduction of a correction coefficient $\gamma$ in the relation of the molecular agitation flux is proposed:

$$
\Phi_{M A}=3 / 2 \gamma C D / \Delta r_{w}
$$

Combining eq. 8 and $9, P_{s}$ is found to write [24]:

$$
\tilde{P}_{s}=\frac{1}{1+\frac{3 \gamma D}{2 k \Delta r_{w}}}
$$

A numerical study is carried out in the validation section to validate the existence of $\gamma$ for the random walk implemented and to assess its value.

For ablation study, the key outputs are the local surface recession, whose fluctuations lead to surface roughness, and the global ablation velocity. Since the molar volume of the material is low compared to the gas, the surface evolution is slow compared to gas diffusion. In actual experiments, a huge number of reactant molecules may be necessary to gasify the volume of one elementary cube of solid. Using numerical simulation, it is impossible to have such a fine description, which fortunately is in no way necessary, especially in view of the discrete mode of geometry representation [19]. Then, the number of walkers can be restricted, using for each walker a representativeness of many reactant molecules estimated as follows. An elementary cube is assumed to be ablated when the corresponding number of sticking events reaches a given number $N$. N has to be chosen large enough so that statistical fluctuations are small, but is otherwise arbitrary. Theoretically, the statistical precision is roughly given by $N^{-1 / 2}$; a practical value for $N$ is then higher than 100 [19]. The computational time being approximatively proportional to $N$, the interest of choosing $N$ as low as possible is obvious. A parametrical study is also useful here to optimize the computational time, without decreasing the accuracy of the result. This is addressed in the next subsection.

\subsection{AMA validation}

The first step is to try and find a value for $\gamma$. Let us use for this purpose the classic 1-D steady state diffusion/reaction model [24], which can be reduced from the general model proposed in section 2.1. The 1D reactant flux $(\Phi)$ at the interface can be analytically obtained [6] and writes:

$$
\Phi=\frac{D}{\delta_{c}} C_{0} \frac{1}{1+\frac{1}{D a}}
$$

where $D a=k \delta_{c} / D$ is a Damköhler number. The evolution of the flux as a function of the Damköhler number is illustrated in figure 3-b. For small Damköhler numbers, the flux is not a function of the reactivity (or of $\gamma$ ); this regime is called diffusion limited. At the other limit ( $D a \gg 1$ ), the consumed flux is 
only a function of the reactivity and the boundary layer concentration. A parameter variation study on $\gamma$ in reaction limited regime ( $D a=0.001)$ is presented figure 3-a. It shows that if $\gamma$ exists its value must be 0.8 . In the reaction limited regime, the normalized flux must display a variation proportional to $1 / \gamma$ (from equations 11 and 10). This is verified and shows the existence of $\gamma$. The two percent uncertainty in reaction limited regime is attributed to the Simplified Marching Cube description of the surface that saves computational time but is only a linear approximation of the interface position, leading to a slight error on the estimation of the surface area. Using $\gamma=0.8$, a parameter variation is carried out on $D a$. As shown in figure 3-b, a correct agreement is obtained for all reaction-diffusion regimes. The uncertainty is found to be lower that the symbol size for any value of $D a$. This comes from the fact that the uncertainty decreases with $D a$ because the diffusion process, which progressively dominates with the increase of $D a$, is independent of the local surface description.

Random walks have the particularity to converge on average at a rate proportional to the square root of the number of random drawings. The convergence has been obtained for $\Delta t_{e} \geq 100 \Delta t$ for diffusion and $N \geq 10$ for reaction. In further simulations, these numerical conditions are systematically implemented using a preprocessor. Increasing the ratio between $\Delta t_{e}$ and $\Delta t$, that is, decreasing $\Delta r$ and increasing $N$, would lower the amplitude of the oscillations, but would dramatically enhance the computing time without changing the average value. For example, for a cubic cell of 100 pixel side length, it is more efficient and valid to use the average flux as a reference value, using the following numerical parameters: $\Delta t_{e}=100 \Delta t$, and $N=10$.

The quality of the moving interface description has also to be checked in 3D. The model of section 2.2 has been solved using AMA, in all regimes for $A=5$ (see figures 1-c where $S h<<1$, and 4-bottom). These results are in qualitative agreement with the analytical results of figures 1-b, and 4-top respectivelly. To enable a quantitative validation, sections of the analytical (black line) and of the numerical solutions (grey-filled part) are compared at figure 5. The overall agreement between the two solutions is excellent: the error is not larger than one pixel width. In the computed cases, the average horizontal section of the numerical cells is around one hundred square pixels, which is a correct compromise between computational time and the quality of the interface description. The computational time is about 24 hours with a $3.2 \mathrm{GHz}$ CPU computer. 


\section{Application to ablation}

At microscopic scale, some roughness features take place on the stripped and pointed ablated fibers. In particular, ex-PAN fibers of 3D C/C composites have been shown to be roughly conical but also faceted after ablation $[6,25]$. On figure 6-c, some fibers from a 3D C/C oxidized under dry air at atmospheric pressure show such facets. The oxidation process as well as the composite are fully described in [7]. The ablation regime is shown to be reaction-limited $(S h<<1)$ [6]. In this case, when steady state is reached, fibers with circular cross-section display a regular conical shape (see figures 1-b and c). Nevertheless, SEM (Scanning Electron Microscopy) analyses of the fibers cross-section clearly show that it is irregular (see figure 6-a). This may have an effect on the steady state geometry and behavior of the fibers. To verify this hypothesis, the irregularities of the fibers cross-section have been introduced in the model presented in section 2.2, as represented on figure 6-b. The steady-state result of the simulation is presented on figure 6-d ${ }^{1}$. As a whole, the large facets which are experimentally observed on the fibers (from fiber tip to bottom) are qualitatively well reproduced. The numerical approach thus proves that they are originated from the irregularities of fibers sections. However, some facets, which are smaller, cannot be explained by this model. They may be linked to anisotropic nature of the graphitic carbon microstructure [26]. Indeed, a modified version of AMA accounting for anisotropic, orientation-dependent reactivity has allowed to show that the microstructure of a polycrystalline graphite acquires under ablation a characteristic roughness linked to its grain distribution [26].

\section{Conclusion}

A simulation tool useful for the modeling of surface roughness onset during ablation has been introduced in its context, presented, and validated. First, a general model for receding surfaces under a first-order gasification process with a mass transport controlled by diffusion, which has been shown to explain well the ablation phenomenon, has been presented. To solve this model in the general case, numerical simulation tools are useful. AMA, which an efficient solution to solve reaction/diffusion problems with a receding surface based on a 3D numerical simulation code developed on a Monte-Carlo Random-Walk principle with a Simplified Marching Cube surface discretization scheme, has been presented. AMA

$\overline{1}$ A movie showing the transient regime of ablation, from a flat surface to the steady-state regime, is provided online (see Elsevier website) 
includes an automatic step refinement close to the wall that lowers the computational time. The reactivity is simulated using a sticking probability adapted to the random walk used (Brownian motion simulation technique). The resulting interface evolution in $3 \mathrm{D}$ has been validated by comparison to an analytical model obtained in a previous study. As an application, the ablation of cylindrical fibers with a noncircular basis, embedded in a more reactive matrix, has been studied using AMA. An interpretation of the origin of the large facets experimentally observed on sharp ablated fibers has been proposed. The large facets basically arise from the irregularities of fibers section which are intensified by ablation. This numerical simulation tool is then useful and efficient to analyse and understand surface roughness onset during ablation of composite materials.

As an opening, this code could be used as well to simulate other phenomena related to mass loss or mass deposition, including for example crystal growth, surface coating processes, sedimentation, and clogging that involve a similar physics but with a growing wall instead of a receding surface.

\section{Acknowledgments}

The authors wish to thank Y. Aspa (LCTS-IMFT), J.-F. Epherre, J.-M. Goyhénèche, and G. Duffa (CEA) for fruitful discussions. The authors also wish to thank CNRS and CEA for a Ph. D. grant to J. Lachaud.

\section{References}

[1] E. Fitzer, L. M. Manocha, Carbon reinforcements and C/C composites, Springer, 1998, 342 p.

[2] G. Duffa, G. L. Vignoles, J.-M. Goyhénèche, Y. Aspa, Ablation of C/C composites: Investigation of roughness set-up from heterogeneous reactions, International Journal of Heat and Mass Transfer 48 (16) (2005) 3387-3401.

[3] K. Kuo, S. Keswani, A comprehensive theoretical model for carbon-carbon composite nozzle recession, Combustion Science and Technology 42 (1986) 177-192.

[4] S. Pestchanyi, V. Safronov, I. Landman, Estimation of carbon fibre composites as ITER divertor armour, Journal of Nuclear Materials 329-333 (2004) 697-701. 
[5] M. R. Wool, Summary of experimental and analytical results, Tech. Rep. SAMSO-TR-74-86, Passive Nosetip Technology Program (PANT) (January 1975).

[6] J. Lachaud, Y. Aspa, G. L. Vignoles, Analytical modeling of the steady state ablation of a 3D C/C composite, Int. J. of Heat and Mass Transfer 51 (9-10) (2008), 2618-2627.

[7] J. Lachaud, N. Bertrand, G. L. Vignoles, G. Bourget, F. Rebillat, P. Weisbecker, A theoretical/experimental approach to the intrinsic oxidation reactivities of $\mathrm{C} / \mathrm{C}$ composites and of their components, Carbon 45 (2007) 2768-2776.

[8] G. Vignoles, J. Lachaud, Y. Aspa, Roughness evolution in ablation of carbon-based materials: Multiscale modelling and material analysis, European Space Agency Special Publication, 631 (2006), 8 p.

[9] G. Vignoles, J. Lachaud, Y. Aspa, J. M. Goyheneche, Ablation of carbon-based materials: multiscale roughness modeling, to appear in Compos. Sci. Tech.

[10] Y. Aspa, M. Quintard, J. Lachaud, G. L. Vignoles, Identification of microscale ablative properties of C/C composites using inverse simulation. AIAA 2911 (2006) 52-64.

[11] G. L. Vignoles, O. Coindreau, A. Ahmadi, D. Bernard, Assessment of geometrical and transport properties of a fibrous $C / C$ composite preform as digitized by $\mathrm{X}$-ray computed micro-tomography. Part II : Heat and gas transport, J. Mater. Res. 22 (6) (2007) 1537-1550.

[12] I. C. Kim, S. Torquato, Determination of the effective conductivity of heterogeneous media by brownian motion simulation, J. Appl. Phys. 68 (8) (1990) 3892-3903.

[13] M. M. Tomadakis, S. V. Sotirchos, Ordinary, transition and Knudsen regime diffusion in random capillary structures, Chem. Eng. Sci. 48 (19) (1993) 3323-3333.

[14] M. Plapp, A. Karma, Multiscale finite-difference-diffusion-monte-carlo method for simulating dendritic solidification, J. Comput. Phys. 165 (2000) 592-619. 
[15] W. E. Lorensen, H. E. Cline, Marching cubes: A high resolution 3D surface construction algorithm, in: M. C. Stone (Ed.), SIGGRAPH '87 Proceedings, Vol. 21 of ACM Computer Graphics, ACM Press, New York, 1987, pp. 163-169.

[16] I. V. Katardjiev, G. Carter, M. J. Nobes, S. Berg, H.-O. Blom, Three-dimensional simulation of surface evolution during growth and erosion, Journal of Vacuum Science and Technology A 12 (1) (1994) 61-68.

[17] J. Lachaud, Simulation of ablation of carbon-based composites, $\mathrm{PhD}$ thesis 3291, Bordeaux University, France, in English (2006).

[18] S. Torquato, I. Kim, Efficient simulation technique to compute effective properties of heterogeneous media, Appl. Phys. Lett. 55 (1989) 1847-1849.

[19] J. Sallès, J. F. Thovert, P. M. Adler, Deposition in porous media and clogging, Chemical Engineering Science 48 (1993) 2839-2858.

[20] G. L. Vignoles, Modelling binary, Knudsen, and transition regime diffusion inside complex porous media, J. Phys. IV France C5 (1995) 159-166.

[21] A. Gil, J. Segura, CA3D: a Monte Carlo code to simulate 3D buffered diffusion of ions in submembrane domains, Computer Physics Communications 136 (2001) 269-293.

[22] G. Federici, M. Mayer, G. Strohmayer, V. Chuyanov, C. Day, Modelling of deposition of hydrocarbon films underneath the divertor and in the pumping ducts of ITER, Journal of Nuclear Materials 337-339 (2005) 40-44.

[23] A. S. Kim, H. Chen, Diffusive tortuosity factor of solid and soft cake layers: A random walk simulation approach, Journal of Membrane Science 279 (1-2) (2006) 129-139.

[24] J. Lachaud, G. L. Vignoles, J. M. Goyhénèche, J. F. Epherre, Ablation in C/C composites: microscopic observations and 3D numerical simulation of surface roughness evolution, Ceramic 
Transactions 191 (2006) 149-160.

[25] J. C. Han, X. D. He, S. Y. Du, Oxidation and ablation of 3D carbon-carbon composite at up to $3000^{\circ} \mathrm{C}$, Carbon 33 (4) (1995) 473-478.

[26] J. Lachaud, Y. Aspa, G. Vignoles, J.-M. Goyhénèche, 3D modeling of thermochemical ablation in carbon-based materials: effect of anisotropy on surface roughness onset, European Space Agency Special Publication 616 (2006), 10 p.

\section{List of Figures}

1 Validation of AMA by comparison to a 3D analytical model in reaction-limited regime $(A=5)$

2 Schematic description of AMA

3 AMA validation by comparison to a 1-D model

$4 \quad$ Fiber morphology at steady state as a function of $S h$ (with $A=5$ ) : qualitative comparison of analytical solution (top) with AMA simulation (bottom)

5 Ablated fiber sections for different reaction/diffusion regimes: comparison of analytical (black lines) and numerical (grey-filled parts) results

6 Faceted fibers : comparison of experimental observations with a model of irregular fiber section 


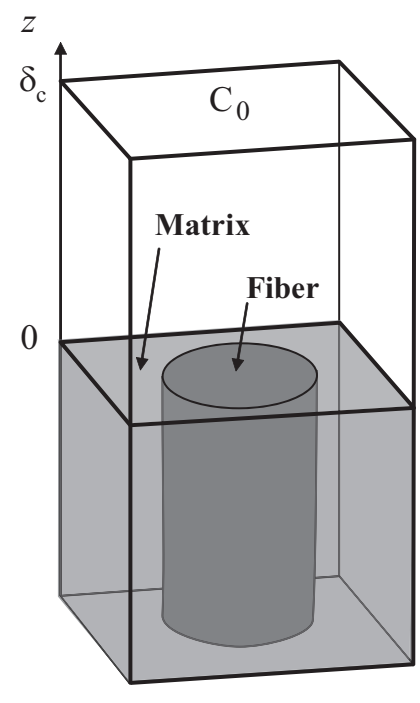

a) 3D elementary cell : initial time

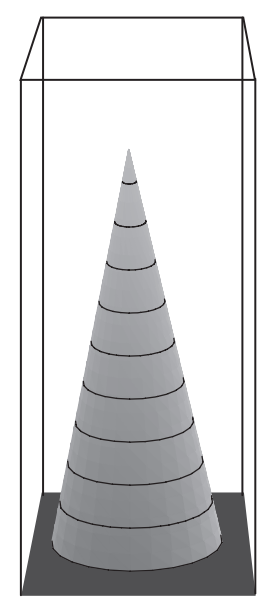

b) Analytical result : steady state

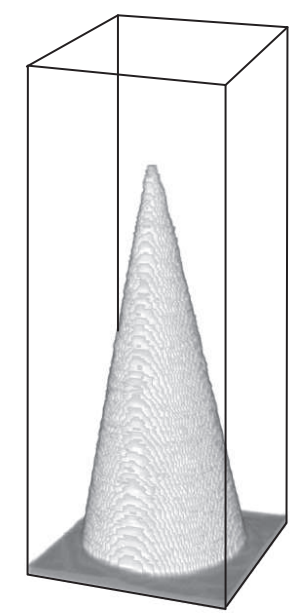

c) AMA result : steady state

Fig. 1. Validation of AMA by comparison to a 3D analytical model in reaction-limited regime $(A=5)$. 


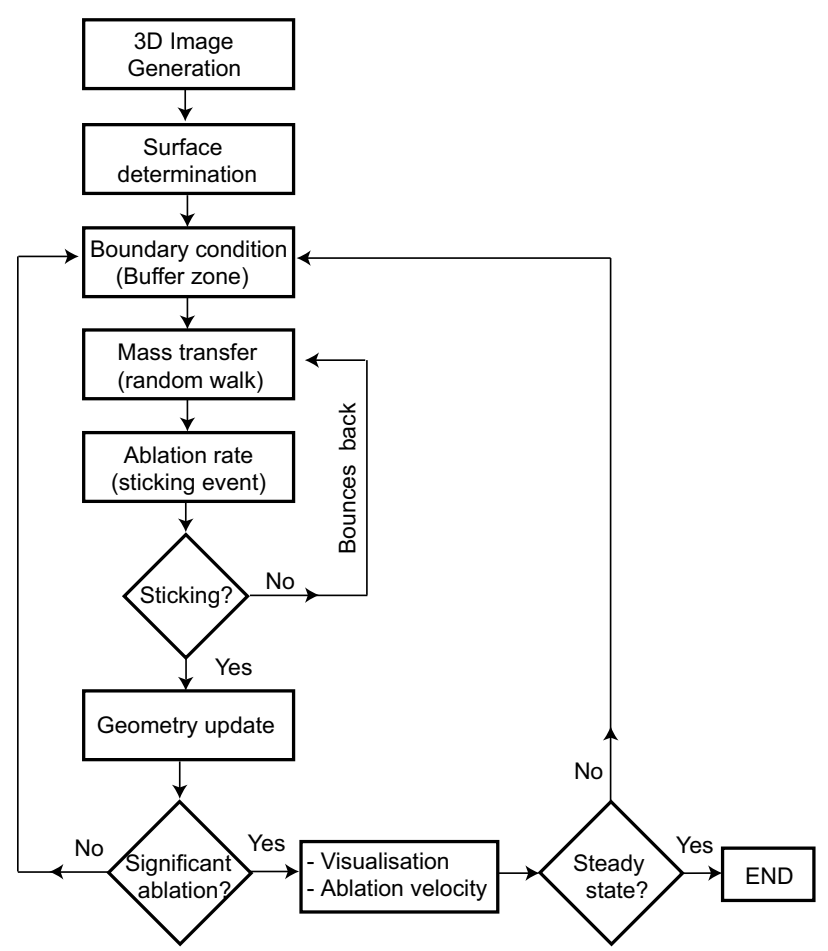

a) Scheme of AMA algorithm

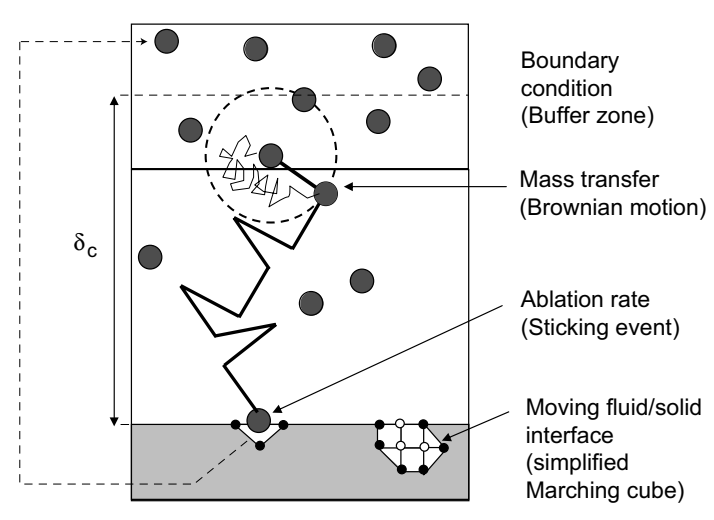

b) Sketch of AMA principle

Fig. 2. Schematic description of AMA 


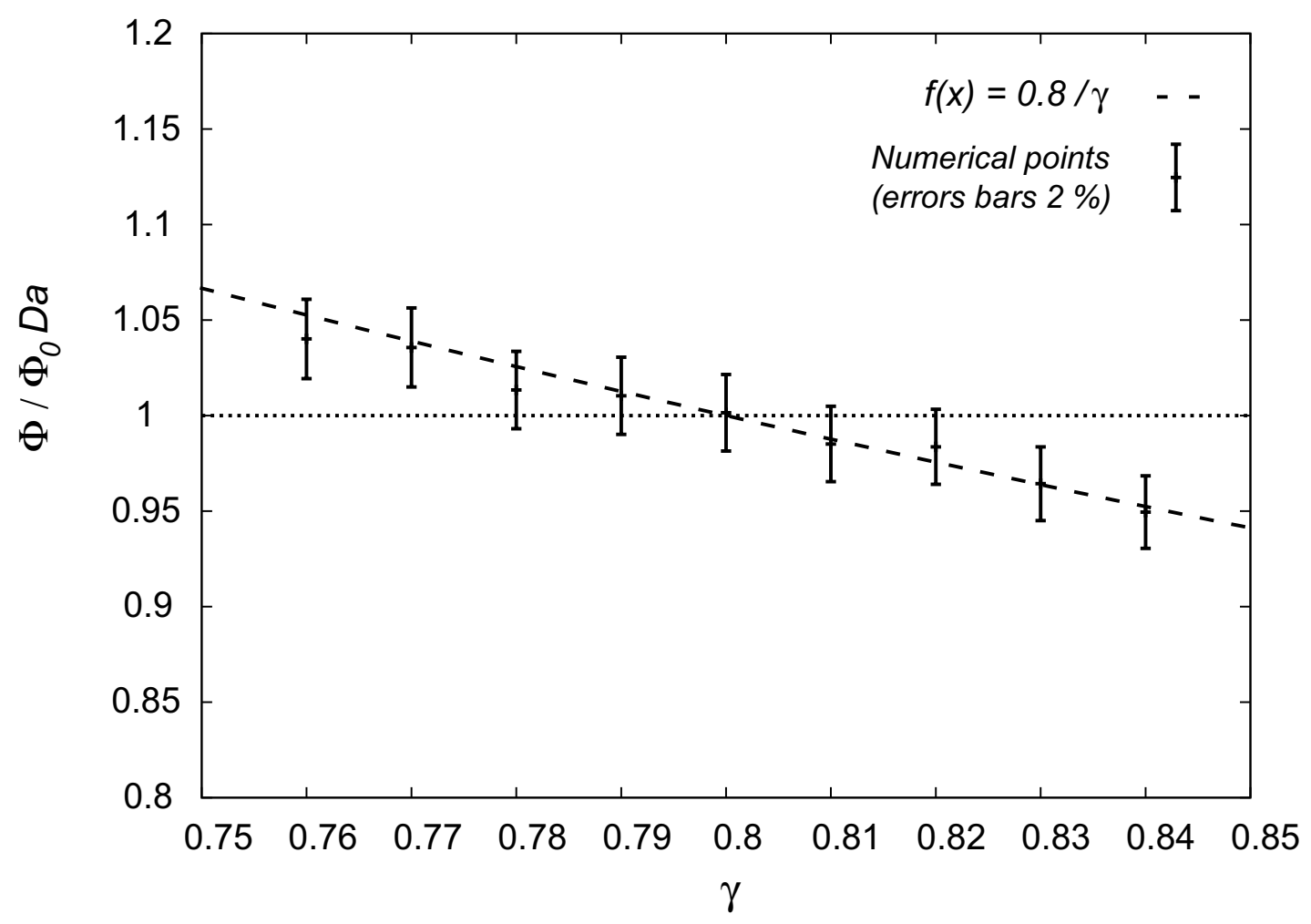

a) Parameter variation study on $\gamma$ in reaction limited regime $(D a=0.001)$

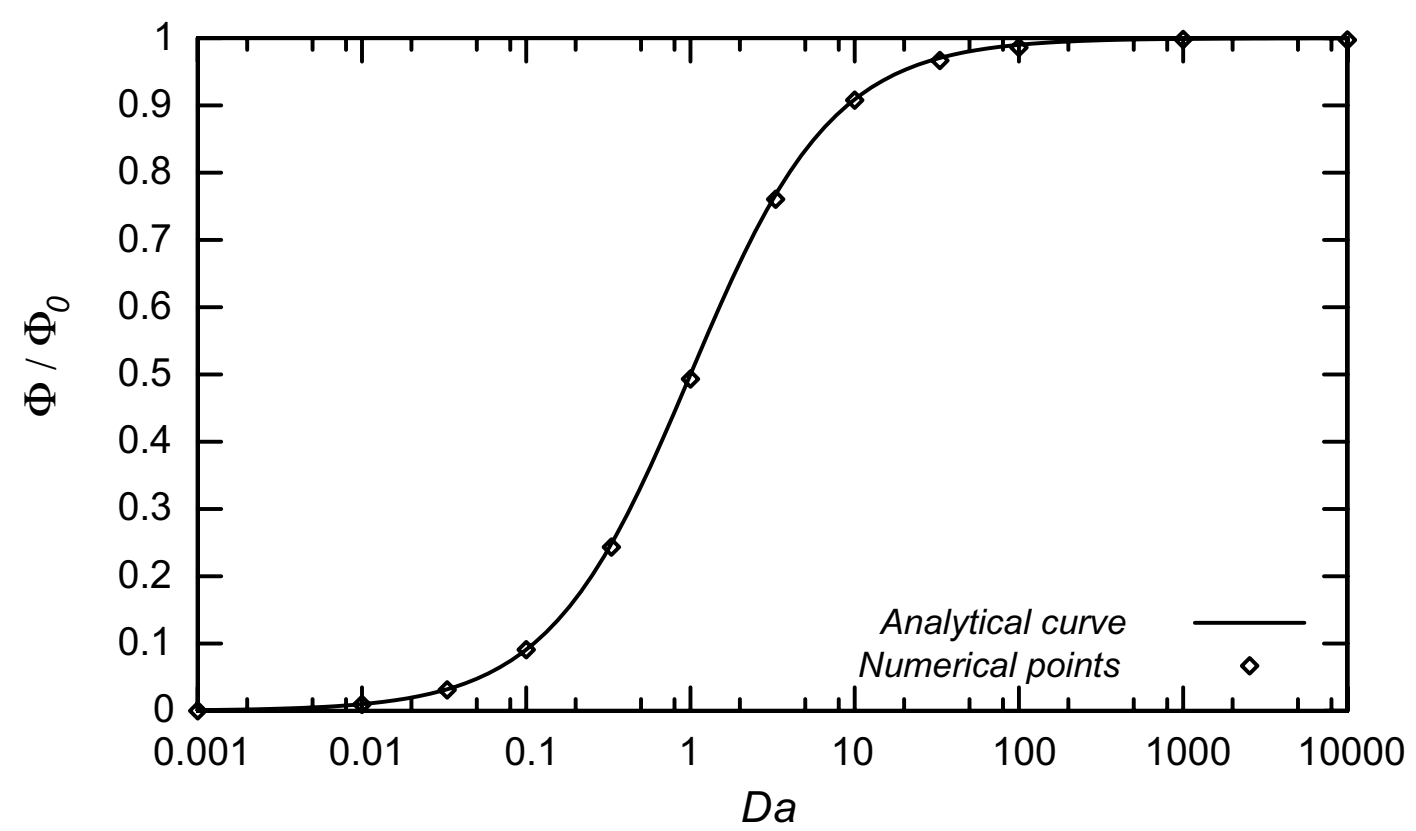

b) Parameter variation study on Da for $\gamma=0.8$

Fig. 3. AMA validation by comparison to a 1-D model $\left(\Phi_{0}=D C_{0} / \delta_{c}\right)$ 


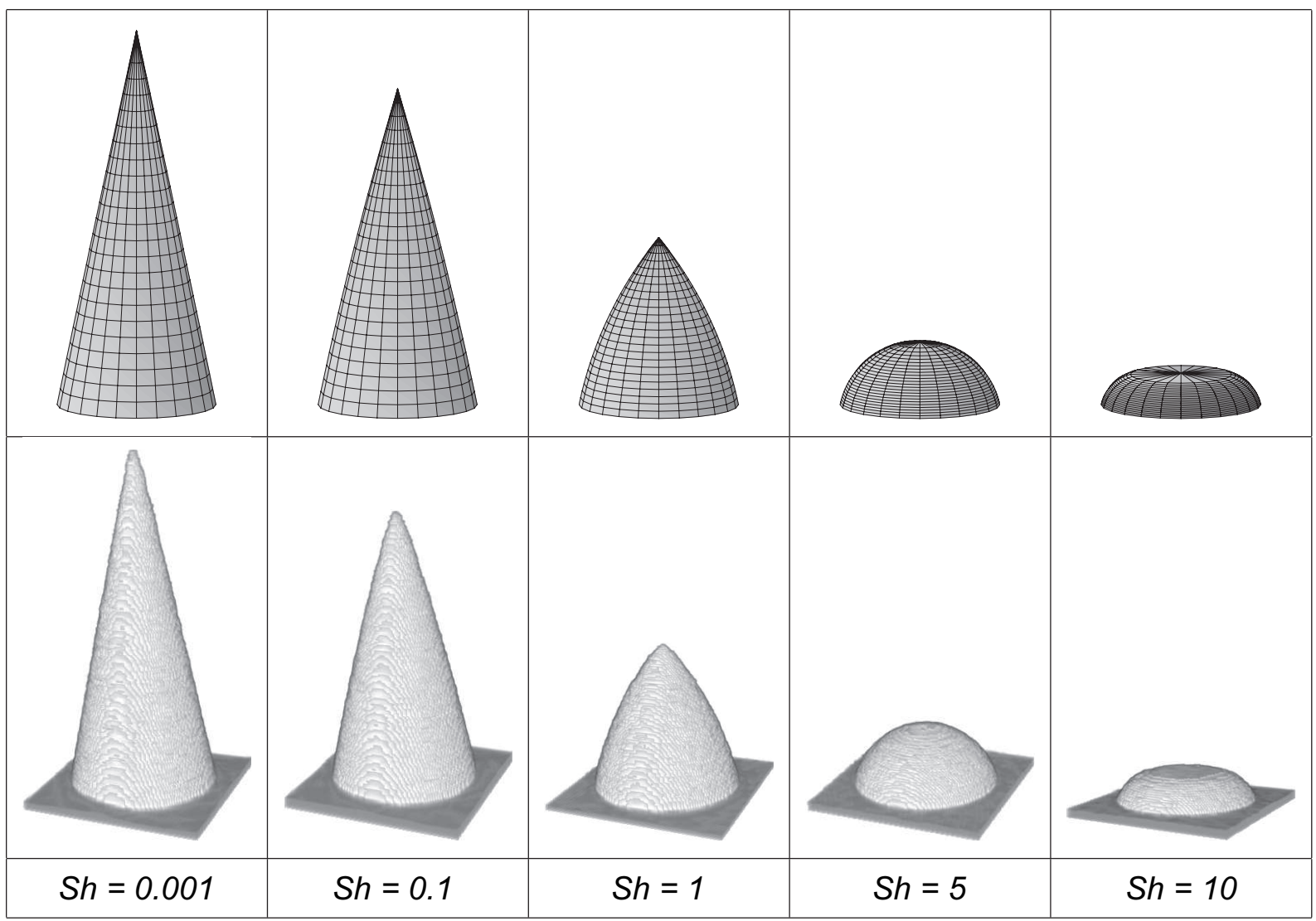

Fig. 4. Fiber morphology at steady state as a function of $S h$ (with $A=5$ ) : qualitative comparison of analytical solution (top) with AMA simulation (bottom). 


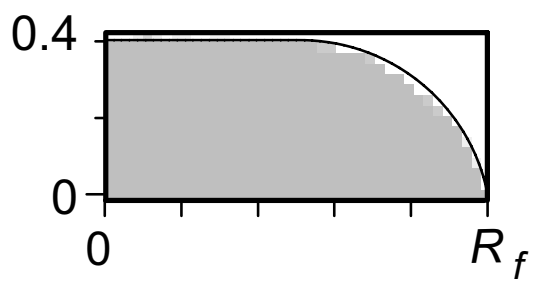

a) $S h=10$

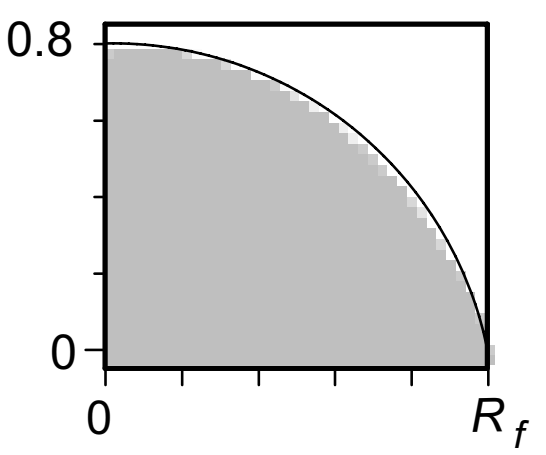

b) $S h=5$

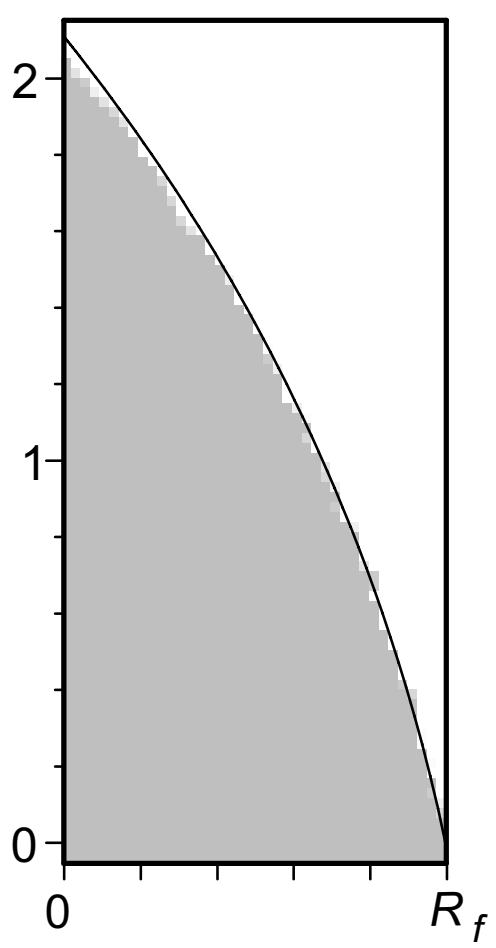

c) $S h=1$

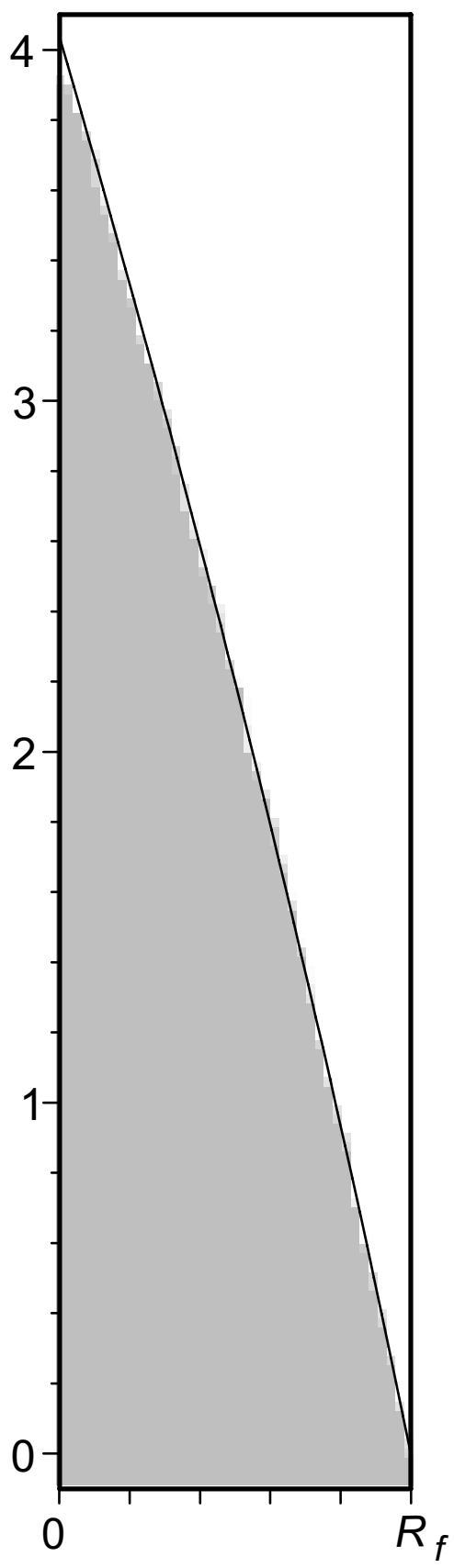

d) $S h=0.1$
5

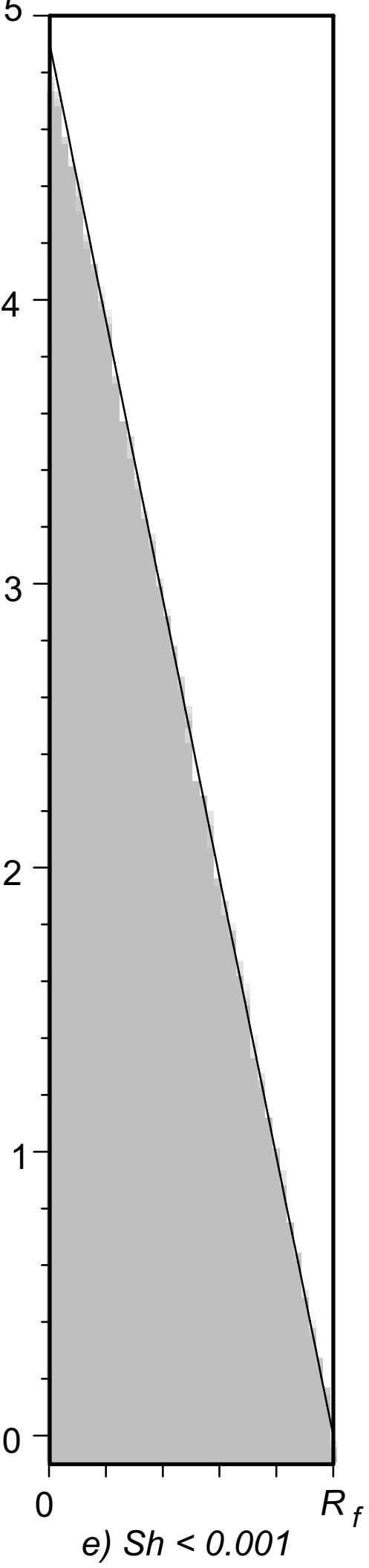

Fig. 5. Ablated fiber sections for different reaction/diffusion regimes: comparison of analytical (black lines) and numerical (grey-filled parts) results. 


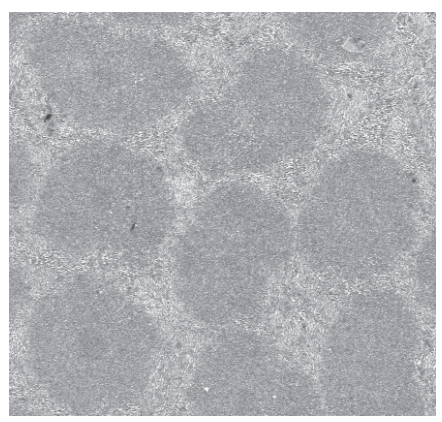

a) Fibers sections : observation (SEM)

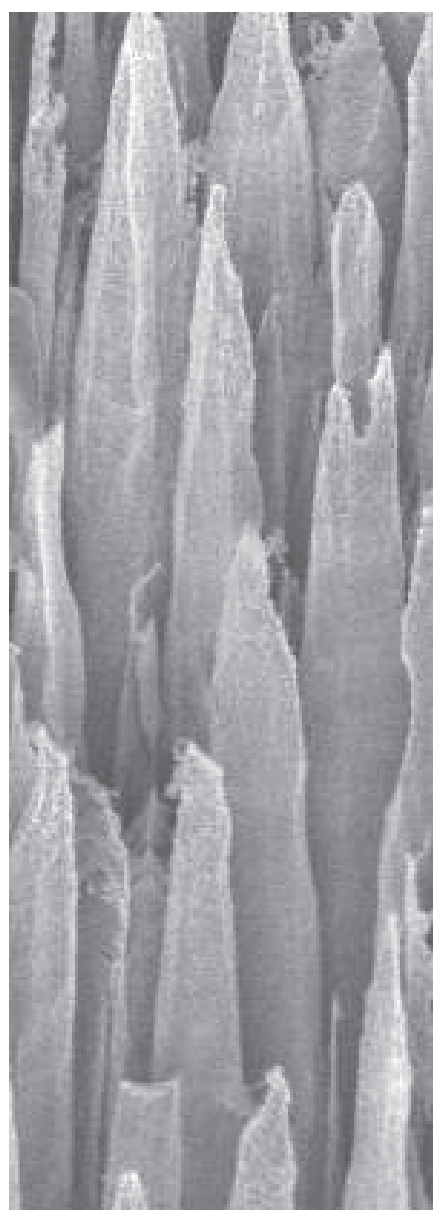

c) Steady state (SEM)

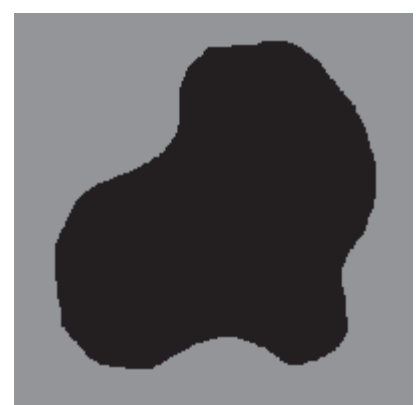

b) Fiber section : model (AMA)

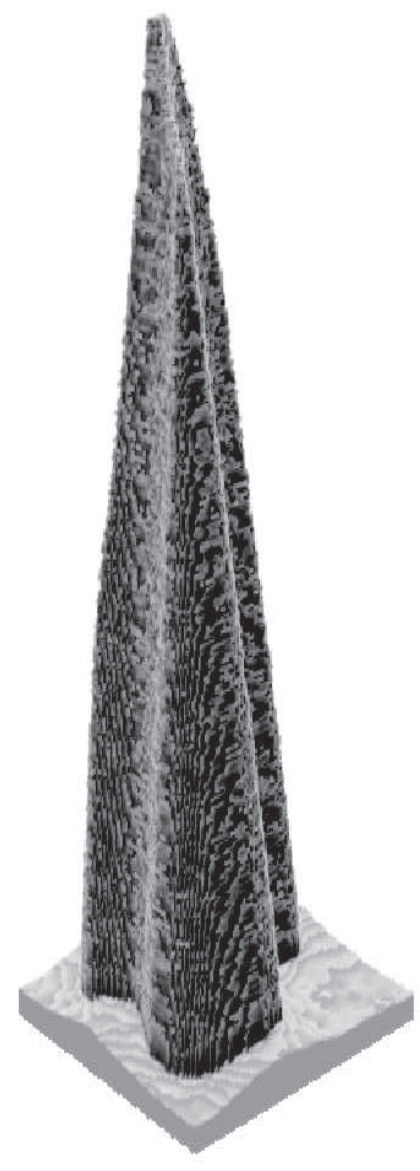

d) Steady state (AMA)

Fig. 6. Faceted fibers : comparison of experimental observations with a model of irregular fiber section 